\title{
Pericarditis due to Bacteroides melaninogenicus secondary to a teratoma
}

\section{TORBEN RIIS RASMUSSEN, JOHN STAGSTED SØRENSEN}

\author{
From the Departments of Cardiology and of Thoracic and Vascular Surgery, Hospital of Aalborg, Aalborg, \\ Denmark
}

Over the past 50 years the aetiology of purulent pericarditis has changed. Most cases used to be caused by Gram positive bacteria, but now there is a higher incidence of cases due to Gram negative bacteria and fungi. ${ }^{1-3}$ Anaerobes are rarely found. ${ }^{3}$ In the following case Bacteroides melaninogenicus, an anaerobic Gram negative bacillus, caused a purulent pericarditis secondary to a perforating teratoma.

\section{Case report}

A 17 year old girl was admitted with signs and symptoms of acute pericarditis with tamponade. Apart from an upper respiratory tract infection 8-10 days earlier she had been well.

The chest radiograph showed a small pleural effusion and an enlarged cardiac silhouette. An echocardiogram showed a pericardial effusion and about $250 \mathrm{ml}$ fluid was subsequently removed at pericardiocentesis. Culture of the fluid yielded an anaerobic Gram negative bacillus after three days' incubation. This was later identified as B melaninogenicus and found to be sensitive to metronidazole but resistant to penicillin.

The patient was treated with intravenous metronidazole, $500 \mathrm{mg}$ thrice daily, for 12 days and her condition gradually improved. Nineteen days after admission, however, signs of constrictive pericarditis developed and a total pericardiectomy was performed.

At operation a cystic tumour measuring $8 \times 6 \times 5 \mathrm{~cm}$ was found in the right mediastinum. It was attached to the thymus and communicated with the pericardium. The tumour, thymus, and pericardium were excised. On histological examination the tumour was found to be a teratoma; there was no evidence of malignancy.

The postoperative period was uneventful.

\section{Discussion}

This is the first report of isolation of $B$ melaninogenicus from a case of purulent pericarditis. This organism is a normal

Address for reprint requests: Dr T R Rasmussen, Banegaardsgade 9, DK-8000 Aarhus C, Denmark.

Accepted 23 September 1987 inhabitant of the upper respiratory tract. It is slow growing, requiring 72 hours' incubation for visible growth, and will be missed if cultures are incubated for only 40-48 hours.

The pathogenesis of the present case, in which a mediastinal teratoma perforated into the pericardial sac, is very unusual. Mediastinal teratomas, accounting for $10-20 \%$ of all tumours of the mediastinum in children and young adults ${ }^{4}$ have been estimated to perforate into the pericardium in less than $1 \%$ of cases. ${ }^{5}$ Formerly such events were diagnosed at necropsy.

The first report of a successfully treated case was published in $1966 .{ }^{5}$ Since then three rather similar cases have been described, ${ }^{6-8}$ though in none of these were bacteria or fungi identified in the contents of the pericardial sac.

\section{References}

1 Rubin RH, Moellering RC Jr. Clinical, microbiologic and therapeutic aspects of purulent pericarditis. $A m \mathrm{~J} \mathrm{Med}$ 1975;59:68-78.

2 Gould K, Barnett JA, Sanford JP. Purulent pericarditis in the antibiotic era. Arch Intern Med 1974;134:923-7.

3 Klacsmann PG, Bulkley BH, Hutchins GM. The changed spectrum of purulent pericarditis. An 86 year autopsy experience in 200 patients. Am J Med 1977;63:666-73.

4 Adebonojo SA, Nicola ML. Teratoid tumors of the mediastinum. Am Surg 1976;42:361-5.

5 Marsten JL, Cooper AG, Ankeney JL. Acute cardiac tamponade due to perforation of a benign mediastinal teratoma into the pericardial sac. Review of cardiovascular manifestations of mediastinal teratomas. $J$ Thorac Cardiovasc Surg 1966;51: 700-7.

6 Aravanis C, Papastreriades E, Steriotis J. Recurrent pericarditis due to cystic teratoma of the mediastinum: a case report. Angiology 1980;31:427-30.

7 Paterson IM, Cockburn JS. Acute pericarditis due to perforation of a benign mediastinal teratodermoid into the pericardial sac. Thorax 1982;37:863-4.

8 Sano S, Shimizu Y, Shimizu N, et al. 'Acute cardiac tamponade due to perforation of a benign mediastinal teratoma into the pericardial sac' [English summary]. Nippon Kyobu Geha Gakkai Zasshi (Tokyo) 1983;31:527-32. 